\title{
Drinking as routine practice among re-integrating National Guard and Reservists from Arkansas
}

\author{
Traci H. Abraham, ${ }^{1,2}$ Ann M. Cheney, ${ }^{3}$ Geoffrey M. Curran, ${ }^{4,5}$ Karen L. Drummond ${ }^{1,2}$ \\ ${ }^{1}$ South Central Mental Illness Research, Education, and Clinical Center (MIRECC), Central Arkansas Veterans Healthcare System; \\ ${ }^{2}$ Department of Psychiatry, University of Arkansas for Medical Sciences, Little Rock; ${ }^{3}$ Department of Social Medicine \& Population \\ Health, Center for Healthy Communities, SOM, University of California, Riverside, CA; ${ }^{4}$ Department of Pharmacy Practice, College \\ of Pharmacy and Division of Health Services Research, Department of Psychiatry, University of Arkansas for Medical Sciences; \\ ${ }^{5}$ Central Arkansas Veterans Healthcare System, North Little Rock, AR, USA
}

\begin{abstract}
Active duty enlisted military personnel are more likely to misuse alcohol than civilians - a pattern which persists even after the transition to civilian life. We used in-depth, substance use history interviews to elicit drinking trajectories from 54 re-integrating Army National Guard, Air Force Reserve and Army Reserve personnel from Arkansas with a history of problematic substance use. A hybrid inductive-deductive analytic approach revealed institutional norms, shared beliefs about drinking, and social values and expectations among military peers present in the context of military service that Veterans described as having shaped their drinking trajectories. Framing Veterans' narratives vis-à-vis practice theory revealed the complex processes by which excessive drinking was embodied as routine practice during military service and subsequently reproduced in a very different post-deployment context, often with deleterious results. Elucidating these implicit processes suggested pro-active strategies for preventing problematic drinking by active duty personnel and improving the re-integration experiences of Veterans.
\end{abstract}

Correspondence: Traci H. Abraham, Central Arkansas Veterans Healthcare System, 2200 Fort Roots Drive, Building 58, Suite 305B, North Little Rock, AR 72114-1706, USA.

Tel.: 507-257-1744

E-mail: Traci.Abraham@va.gov

Key words: Practice theory; military culture; veterans; alcohol consumption.

Conflict of interest: The authors declare no conflict of interests.

Funding: This research was supported by the Department of Veterans Affairs Office of Academic Affiliations Advanced Fellowship Program in Mental Illness Research and Treatment, the Medical Research Service of the Veterans Affairs of the Central Arkansas Veterans Healthcare System, and the Department of Veterans Affairs Center for Mental Healthcare and Outcomes Research. The VA Clinical Science Research \& Development grant ICX000452A supported the research. The views expressed in this article are those of the authors and do not necessarily reflect the position or policy of the Department of Veterans Affairs or the U.S. government.

Acknowledgements: The authors would like to thank the Veterans who participated in this study and who have reviewed this manuscript for authenticity. Your service to research helps inform meaningful improvements to healthcare that make a difference in the lives of Veterans.

Received for publication: 7 April 2020.

Accepted for publication: 9 September 2020.

This work is licensed under a Creative Commons Attribution NonCommercial 4.0 License (CC BY-NC 4.0).

${ }^{\circ}$ Copyright: the Author(s), 2020

Licensee PAGEPress, Italy

Qualitative Research in Medicine \& Healthcare 2020; 4:43-53

doi:10.4081/qrmh.2020.9001

\section{Introduction}

Active duty, enlisted personnel in the U.S military use alcohol more often than cigarettes or smokeless tobacco (84.5\%, 49.2\% and 19.8\%, respectively). Alcohol consumption far exceeds the use of recreational drugs among active duty personnel. ${ }^{1}$ Levels of drinking nevertheless vary by military branch, with personnel enlisted in the Marine Corps, Army and Navy than those enlisted in the Air Force reporting higher rates of alcohol consumption., ${ }^{2,3}$

We define problematic drinking as a spectrum of alcohol consumption involving risky and excessive drinking (i.e., drinking which increases the risk of poor health outcomes), alcohol abuse, and alcohol dependence. Qualitative and mixed-method research situates active duty personnel problematic drinking in the context of daily life on military base, describing the influence of occupational or military culture on drinking patterns..$^{4-6}$ Ames et al.' ${ }^{7}$ work underscores cultural norms that equate the use of alcohol with stress relief and perceive drinking as a mechanism for social bonding as contributing to higher rates of drinking in the military. Likewise, Larson et al.'s $\mathrm{s}^{8}$ review of combat deployment and alcohol misuse highlights that an indoctrination into military culture that instils attitudes and beliefs about alcohol consumption shapes problematic drinking during deployment. A more recent study concluded that military socialization processes influence the current high use of alcohol among active duty personnel. ${ }^{9}$

Although military culture has often been evoked in the scientific literature to explain problematic drinking in the military, it remains unclear how military culture 
relates to alcohol consumption among Veterans. Evidence indicates that active duty personnel's problematic drinking continues as they transition to civilian life ${ }^{10}$ and that rates of alcohol use disorder (i.e., compulsive alcohol consumption and loss of control over the volume of alcohol consumed, accompanied by a negative emotional state when not using alcohol) is high among more recent Veterans. ${ }^{11}$ As some studies have reported rates of alcohol misuse as high as $40 \%$ among ${ }^{12}$ Veterans of recent U.S. conflicts, ascertaining the role of military culture in the perpetuation of problematic drinking is particularly urgent. It is critical to identify innovative and effective strategies for treating and preventing problematic drinking in Veterans.

In this study, we aimed to gain an understanding of substance use trajectories among Veterans for whom consuming substances (i.e., alcohol, illicit drugs) had complicated their re-integration experiences. We used snowball sampling to recruit 45 males and seven females $(\mathrm{N}=54)$ Veteran and/or active duty National Guard and Reservists from Arkansas with a history of problematic substance use. We collected data using in-depth substance use life history interviews, using a hybrid inductive-deductive coding approach to analyze interview data. During the process of developing analytic memos, we observed concepts from practice theory emerge inductively.

\section{Bourdieu's Practice Theory}

Bourdieu's ${ }^{13,14}$ practice theory provides a framework for explicating how Veterans' routine health behaviors develop and are reproduced (i.e., maintained over time) through daily interactions and practices. This framework lends valuable insights into the implicit processes that contribute to Veterans' problematic drinking. According to Bourdieu, all behavior is set in social arenas called fields, or contexts. ${ }^{15}$ Cooper et al. ${ }^{16}$ describe the military as a context distinct from the varied collection of alternative fields which constitute civilian life. This context is nested, consisting of the military as an institution and different arenas/contexts subsumed within that institution (e.g., the regiment). Regardless of military branch, new recruits are immersed within the physical boundaries of a military base possessing its own internal logic, set of rules and regulations, and reference groups.

These contexts generate a habitus, or framework of shared understandings and behavioral dispositions or embodied ways of being. ${ }^{17}$ Habitus determines which behaviors are constituted as socially intelligible, normative and expected. A masculine-warrior habitus, which includes stereotypically masculine traits of self-control, courage, physical and emotional "toughness" (i.e., resilience), competitiveness, and physical aggressiveness structures the U.S. military. These traits, which prepare soldiers for combat, are valued and rewarded. ${ }^{18}$ The process of basic training, which physically and socially isolates new recruits from the civilian world, socializes new recruits in all branches of the military, including National Guard and Reservists, to the military habitus.

By acting in ways that are valued within a social arena or context, social agents accrue capital (i.e., economic resources and social status). In this regard, military personnel who exhibit stereotypically masculine traits are rewarded with respect and loyalty from peers. Superiors also promote them up through the ranks. Thus, military personnel strategically internalize behaviors that will help them accrue capital such as practices to earn respect (i.e., social capital) and be promoted (i.e., economic capital). In the process, they embody habits or behavioral dispositions that reproduce accrued capital. By embodying a habitus, military personnel adapt to external conditions largely beyond their individual control and perform, without thinking, socially and institutionally patterned behaviors routinely.

Structure in the Bourdieusian sense is therefore both external and objective (e.g., social expectations) and internal and subjective (e.g., shared ways of perceiving and understanding). By eliciting or constraining behaviors, structure produces behavioral similarities among social agents within a context - also known as culture. Once embodied, routine practices structured by a habitus are often enduring, and thus uncritically reproduced from one context to another. What's more, although habitus is a produce of social conditioning and thus transforms as social agents transition from context to context, a mismatch can occur between habitus and context as social actors transition from one social arena to another. Bourdieu terms this mismatch hysteresis. ${ }^{13}$ The latter has important implications for how routine practices learned in the military, such as problematic drinking, might be reproduced out of context, and the potentially deleterious effect of reproducing behaviors thusly. Understanding these implicit processes could thus support the development of more effective treatments.

\section{Materials and Methods}

\section{Study design}

We aimed to investigate substance use and re-integration among fifty-four ( $\mathrm{N}=54)$ Army National Guard, Air Force Reserve and Army Reserve personnel from Arkansas; henceforth collectively referred to as Veterans. Substance use refers to consumption of alcohol, prescription drugs and/or illicit drugs. Our multidisciplinary study team consisted of three research investigators, including one PhD-level medical sociologist and three PhD-level medical anthropologists. A research assistant possessing a Master's in public health and minor in anthropology supported the investigators.

Our study design combined rapid-ethnographic techniques that combined insights gained from first-hand observations and informal interviews at military bases with in-depth substance use life history interviews. We selected 
this study design based in the potential for rapid ethnography to elucidate the implicit, contextual processes that contribute to Veterans' problematic drinking and suggest realistic strategies to develop health interventions for this population. Rapid ethnographies use long-standing ethnographic methods (i.e., first-hand observations, in-depth interviews), but focus more narrowly upon a health-related issue (e.g., problematic drinking) and well-defined context (e.g., military service).$^{19}$ As the latter renders context- and experience-rich research feasible in the condensed timelines of health services research, rapid ethnography is increasingly used to develop and assess health interventions. ${ }^{20}$ The Institutional Review Board of the Center for Mental Healthcare and Outcomes Research, Central Arkansas Veterans Healthcare System approved study procedures.

\section{Participant selection}

We recruited potential participants via contacts within the National Guard (e.g., chaplains, family groups, medical personnel) and, in keeping with rapid ethnographic approaches, in-person at National Guard events and Veterans Administration clinics. We also used snowball sampling procedures. ${ }^{21}$ We asked participants to tell their friends about the study and to have them contact a member of our team if they believed that they would meet eligibility criteria.

Immediately before each interview, a member of our team used a modified version of the Alcohol Abuse and Dependence and Non-Alcohol Psychoactive Substance Use Disorders sections of the Mini International Neuropsychiatric Interview (MINI) to screen volunteers for eligibility. The MINI is a short structured diagnostic interview used to identify psychiatric disorders in the past 12 months. ${ }^{22}$ After experiencing recruitment challenges (i.e., volunteers who had not misused substances in the specified time period), we broadened the tool to assess substance use since entering the military. This strategy allowed us to recruit Veterans with diverse substance use trajectories that had nevertheless once lived on a military base, were socialized in the military ethos, and had experienced the transition from the military to civilian life. As Veterans who volunteered were explained the purpose of our study during recruitment and before consenting to participate, the excerpts presented below represent the perspectives and experiences of Veterans who acknowledged having consumed alcohol excessively since having entered the military.

\section{Participants}

A total of 45 males and seven women $(\mathrm{N}=54)$ Veteran and active duty National Guard and Reservists from Arkansas with a history of problematic substance use participated. Participants ranged from 23 to 70 years of age. A majority had served overseas, including deployments to
Afghanistan, Bosnia, Ecuador, Germany, Iraq, Vietnam and Korea. All but one were enlisted military personnel (i.e., not commissioned officers). While some participants initially enlisted in the National Guard or Reserves, others initially enlisted full-time and subsequently joined the Guard or Reserves. Regardless of these differences, all participants had been socialized in the military via basic training, allowing us to identify shared perspectives and experiences that transversed military branch and service era.

\section{Data collection}

We first collected data using focused observations and informal interviews conducted at National Guard drill exercises, deployment preparation briefings, pre-deployment health screenings, day-long post-deployment service events, and events sponsored for service members and families (e.g., retirement ceremonies). These data helped us gain a sense of general attitudes, shared understandings, language, and social practices related to substance use in the National Guard and Reserves. We recorded observations and informal interviews as field notes, using these data to develop interview guide questions for the substance life history interviews (described below) that included terminology familiar to Veterans (i.e., acronyms common in the military).

We then used substance use life history interviews, a form of life history interview adapted to the goals of this study, to collect data pertaining to individual substance use trajectories, perceptions of substance use, and histories of help seeking for substance misuse. Life history interviews permit an in-depth exploration of individual biographies $^{23}$ and are well-suited to embedding individual-level data in social and cultural context. ${ }^{24}$ These interviews employ broad, open-ended questions to explore events in a participant's life history to which he or she retrospectively assign significance, and those experiences influenced behavior in relation to the topic of interest (e.g., substance use). Life history interviews produce indepth narratives with render explicit the meaning that participants attribute to behavior and the contexts in which behavior is situated, producing rich data that is amenable to theory development.

For this study, we used life history interviews to explore substance use in the context of participants' military service and transition to civilian life. Although we used a semistructured interview guide to keep questions consistent across participants, questions were raised in an open a manner to encourage lengthy narratives describing the social context, interpersonal relationships, organizational structures, and individual life experiences that influenced substance use. The semi-structured interview guide is presented in Appendix. Interviews took place face-to-face, in Veterans' homes or in the privacy of staff members' offices, depending on participant preferences. The substance use life history interviews were 90 minutes to four hours in length, and audio recorded with participants' permission. 
Audio recordings of the interviews were transcribed verbatim and imported into a software program for analysis.

\section{Data analysis}

We used a hybrid deductive-inductive approach following the tenants of content analysis to analyze the interview transcripts. ${ }^{25}$ Two anthropologists on our team first developed top-level deductive codes related to the goals of the study (e.g., perceptions of substance use). This initial coding scheme was later deepened by incorporating inductive codes (e.g., military culture) that emerged repeatedly across interviews through an iterative reading of the first batch of transcripts. During subsequent readings of transcripts, we refined the initial coding scheme and developed sub-codes. A software program was used to ensure coding consistency and to help the coders attain intercoder agreement. ${ }^{21}$

The software program's memo feature, which permits analysts to interact more fully with data by reflecting upon the relationship between codes facilitated theory development. Memoing supports the development of hypotheses regarding the relationship of patterns in the data to the context of military service and re-integration. ${ }^{26} \mathrm{~A}$ close reading of individual biographies led to the development of memos referencing key Bourdieusian concepts (e.g., context, adaptation, routine practice). Our use of Practice Theory as a conceptual framework is thus consistent with how participants made sense of their substance use life histories and re-integration experiences.

\section{Results}

During substance use life history interviews, Veteran participants described their socialization and experiences in the military. Qualitative analysis of individual biographies revealed institutional practices and norms, shared beliefs about drinking, and social values and expectations among military peers that participants believed contributed to the development of problematic drinking. Participants also described problematic drinking vis-à-vis structural conditions present on military bases at home and overseas.

\section{The social and institutional context of drinking among active duty personnel}

Veterans consistently recalled that as new recruits they were immersed in a context in which consuming large quantities of alcohol was routine practice (i.e., per Bourdieu, a behavior which is common and accepted uncritically and therefore normative). The normalization of drinking is evident in the words of an Army Veteran who stated that: I think I started drinking before I joined the military around 16, 17 years old. But after I joined the military in '82, I was exposed to it every day (S1031). Accordingly, most participants described having first embod- ied problematic drinking as habitual practice following submersion in the military arena. The contrast between life before and after having been socialized to the military habitus is evident in the following exchange between a researcher and a 23-year-old Veteran of Operation Iraqi Freedom who had been an in-patient for alcohol dependency treatment multiple times:

Interviewer: Before you joined the military, had you used anything, or did you drink at all?

Respondent: I had a baseball scholarship and I was on the swim team. I didn't do drugs, I didn't drink at all; but, you know, it was always around.

Interviewer: It started afterwards?

Respondent: Yes ... yes, it started after. Definitely. Definitely (S1003).

While a small minority remembered drinking casually prior to military service, most participants recalled consuming alcohol routinely and in large quantities only after entering the military. The excerpt which follows illustrates how drinking excessively as habitual practice was embodied during military service. The participant recounted how, during the conflict in Vietnam:

Interviewer: Vietnam was the first time you started drinking?

Respondent: Well, basic [training], really... We got a three-day pass, went down to town, got drunk, throwed up. You know, ever since then [emphasis placed by the authors] (S1016).

In recounting that we...went down to town and got drunk, the Veteran cited above alluded to drinking as a social practice among new recruits, rather than an individual behavior. Likewise, the words ever since then in the same passage renders the habitual nature of drinking as a practice embodied during military service and reproduced by Veterans following re-integration into the civilian context evident. Participants were thus aware of having embodied new routine practices related to alcohol consumption through their socialization in the military habitus, and often explicitly referenced routine drinking as a socially and institutionally patterned behavior.

Veterans furthermore described shared understandings, such as a work hard play hard attitude, present in the military habitus that framed alcohol consumption as a reward for hard work. These shared understandings normalized (and valorized) problematic drinking. In the social arena shaped by this habitus, even frequent, excessive drinking was perceived as unproblematic. The way in which shared understandings elicited drinking as routine practice is evident in the words of a woman Veteran of Operation Desert storm. She stated: The majority of alcoholics and addicts are in the military, and most of them don't know or don't realize that they're alcoholics. Be- 
cause when you get off duty, you go drink. And that was the norm... It's just everyday routine (S1019). By referencing excessing drinking as a routine practice accepted uncritically (i.e., most of them don't realize that they're alcoholics) in this social arena, this Veteran also alludes to the internalization of habitual drinking (i.e., It's just everyday routine). Like many in our study, she also recognized in hindsight that the very ordinariness of drinking among military peers made the routine practice of drinking excessively problematic.

Veterans also explicitly referenced institutional norms (e.g., regulations) that rescinded economic capital from military personnel who consumed other substances (i.e., illicit drugs) and inadvertently promoted or valorized alcohol consumption. These norms included random drug testing and stronger putative measures enforced against those who screened positive for drug use. If you get caught drinking on duty, you're going to get your hand slapped and maybe even get some money taken from you, one Veteran explained. If you get caught doing drugs on duty, you're definitely going to get money taken from you (S1013). Participants also stated that superiors reinforced institutional norms in the military by ignoring or encouraging drinking. In the excerpt below, a Veteran deployed to Egypt and Iraq who had been an in-patient for alcohol dependency two weeks before participating in an in-depth interview wonders how his experience of re-integration might have been different if superiors had not inadvertently encouraged drinking:

And it was very acceptable in the military [to drink]. It still is, yes. Even underage, I mean, it's acceptable. It was a bunch of crap because it should've never been accepted for me. It's almost a shame that it was. I really wish my leaders would've taken a little bit better care of me with that. Because it could be, it could be a little different now, maybe (S1041).

Participants thus attributed the embodiment of drinking as routine practice to military service. Once embodied, problematic drinking was obfuscated by the very routineness of consuming alcohol among military peers, shared understandings in the military regarding what constituted acceptable levels of alcohol consumption, and institutional norms that rescinded economic capital from personnel who consumed other substances.

\section{Drinking during deployment overseas}

While some participants embodied drinking as new recruits and subsequently reproduced that routine practice while deployed overseas, others initially described having begun drinking in the context of deployment. Thus, the way in which the context of military service elicited habitual drinking is evident, as well, in narratives centered on alcohol consumption during deployment. To illustrate, a Veteran of the conflict in Iraq stated: Me and my unit got deployed overseas. Before I went over there, I didn't drink, I didn't smoke, I never even used drugs. My granddad, my uncles were all alcoholics, and it was just never something I ever did (S1027). The words before I went over there, I didn't drink reveals drinking as a practice that had not been embodied before deployment, despite a family history of alcohol abuse.

Veterans moreover often recounted in-depth how the structural conditions present in the context of deployment evoked psychological and emotional distress, and how this structural distress in turn elicited habitual drinking. Participants described structural conditions, including a sense of lacking control, boredom, exposure to physical threats, witnessing deaths and atrocities, and separation from family and other sources of emotional support, as having been present during deployment. Participants often related these conditions, and the structural distress they evoked, to drinking as a routine practice. For example, in the excerpt below, a Veteran describes strategically drinking to manage structural distress experienced while deployed to Iraq:

I kind of was afraid all the time. I had to do my job - that was it. But then once I found out that I can get something to drink over there, hey, I felt better. I'd go to bed, get ready for the next day. You're mentally not there for a minute. That's what drinking did for me (S1014).

The use of alcohol to adapt to the structural distress evoked by contextual conditions present during deployment is evident again in the words of a 44-year-old Veteran who remembered drinking to feel mellow. To ease the pain or ease the mental state - go to bed (i.e., sleep) (S1014) while deployed to Ecuador and Afghanistan. Likewise, another participant described having consumed alcohol habitually to numb everything out (S1034) while deployed to Afghanistan, indicating that drinking was used to cope with unpleasant thoughts and/or emotions and thereby adapt to the context of deployment.

The passages above reveal Veterans as social agents who strategically engaged in a socially valued and institutionally sanctioned practice to adapt to distressing conditions during deployment. Participants thus adapted to structural conditions by reproducing a socially and institutionally valued, routine behavior: habitual drinking. As consuming alcohol, even to excess, was normative and thus perceived as unproblematic by peers and superiors in this context, problematic drinking persisted unchecked if participants could consume alcohol and still perform official duties. To have done otherwise would have violated the values and norms in the masculine-warrior habitus, which rewards combat preparedness with capital.

As alcohol was, in some contexts (e.g., Iraq, Afghanistan), more easily obtained at home than abroad, 
drinking often increased dramatically during combat leave. The danger of having alcohol readily available after having transitioned to the context combat leave is evident in the following statement from a Veteran of the conflicts in Iraq and Afghanistan:

Interviewer: Let's talk about your leave time during deployments.

S1033: I don't remember most of them (i.e., combat leaves). I was drunk. As soon as I got off the plane in Atlanta, I was drinking. I was drunk when I got off the plane, I was drunk when I got back on the plane, and stayed drunk up until we got to Shannon, Ireland for refueling.

Most Veterans in our study described having consumed alcohol excessively during combat leave, sometimes to the point of losing consciousness - repeatedly. What's more, they most often recalled drinking in this context to psychologically and emotionally distance themselves from the structural conditions experienced during deployment. Veterans thus recounted how habitual drinking helped them manage the uncomfortable thoughts and emotions associated with structural distress, and thereby adapt to structural conditions present during deployment, even while on leave.

\section{Habitual drinking post-deployment, during re-integration}

Participants also described having uncritically reproduced drinking habits embodied while in the military in a very different context: civilian life. The way problematic drinking was reproduced uncritically as habitual practice out-of-context is explicit, for example, in the words of a Veteran who described consuming alcohol to excess as just something that I felt like I have to do most of the time (S1014) as a civilian. Other participants described having consumed alcohol habitually after transitioning to the civilian context in a more deliberate manner, often in response to structural distress evoked by this new arena.

Structural conditions described by participants as having been present in the civilian context included a lack of purpose and meaning and everyday life stressors, such as having to pay bills. The different kinds of distress (i.e., stress, anxiety, uncertainty) evoked by the transition from one context to another are evident in the following narrative from a 34-year-old Veteran:

Interviewer: Were there any particular days or experiences that stand out to you during deployment? S1013: A lot of emotions that people deal with that they've never dealt with before and haven't had to deal with the whole time we've been in country (i.e., deployed overseas). But now there's a lot more stress - a lot of different stress. A lot of anxiety, a lot of uncertainty and, you know, you ball that all into the mental psyche where we normally don't deal with things like that very well (S1013).

Thus, having successfully adapted to a highly regimented context with an explicit mission and clearly defined roles, many Veterans in our study were thrust into unfamiliar and distressing circumstances upon transitioning to the arena of civilian life. The depth of distress evoked by the structural conditions present during re-integration is apparent in the words of a Veteran of Operation Iraqi Freedom, who lamented:

Interviewer: So, what was it like to come home when it was finally time to come home - not on leave, but for real?

S1003: How are you happy to be home? How are you happy to come back from killing people? How are you happy to come back from a stress-free life where you didn't have to worry about nothing but waking up, going on missions, coming back home playing video games? I don't know man - it was... different. It was like the first day of school.

For some participants, the very experience of transitioning from one context to another was the primary source of distress. Veterans sometimes explicitly related drinking to the experience of transitioning out of the military context, as illustrated by the following description of transitioning to the civilian context during combat leave:

Interviewer: So, tell me about your leave time.

S1003: Leave, I got fifteen days.

Interviewer: Okay so what did you do? What do people do on leave?

S1003: We got home, man... I trashed so many beer cans and so many bottles. Yeah, man, I got messed up; I'm not going to lie. Whoo! But that's all there is to do. I mean, what are you going to do? I mean, you're transitioning from going to Iraq and fighting all day to coming back home and just supposedly being normal.

Equally distressing for other participants was the way in which the structural conditions present in the context of deployment overseas were reproduced post-deployment. For these Veterans, structural conditions were internalized and embodied post-deployment as lived experiences of trauma. Although no longer deployed for combat, structural conditions were embodied in the form of post-traumatic stress disorder [i.e., PTSD], depression, and survivor's guilt, and thus reproduced in the context of post-deployment civilian life. The embodiment of structural conditions as lived experiences of trauma in the form of survivor's guilt, for example, is apparent in the following passage from an interview with a Veteran of Operation Iraqi Freedom: 
We had one soldier die... He just got back from leave; he had a baby or son, you know, and that was the main thing, just because... I wasn't married. I didn't have any kids, I still don't, you know, and that's -. That's the only thing that ate at me really was like: 'Why not me,' you know? (S1007).

In the expert above, the participant explicitly relates lingering trauma in the form of survivor's guilt (That's the only thing that ate at me really...) to structural conditions encountered during deployment (We had a soldier die). Other participants recounted embodying structural conditions encountered in the context of deployment as moral injury - a profound sense of moral transgression at having witnessed or participated in violence. A Veteran who had been deployed to Iraq described the lived experience of moral injury thusly:

I had two children, so it was like: 'I can't believe I just killed somebody and I hope God forgives me for it,' pretty much. I couldn't sleep for like four days. It took me two days to even eat, man. I mean, I never had killed anybody before I went to the Army. The main thing that really messed with me was my conscious. You always hear what you do unto other people comes back to you... (S1003).

After having internalized a habitus which predisposed them to consume alcohol habitually in response to structural distress, participants thus reproduced that routine practice in response to a different set of structural conditions (e.g., survivor's guilt). Having deliberately consumed alcohol to manage the distress evoked by the structural conditions reproduced as lived experience is evident in the excerpt below from an interview with a Veteran of the conflict in Iraq:

It was a party for six months long. Drugs and alcohol. That's all it was, was a party... I was numbing myself from that feeling of what I've had to do (during deployment). What I've had to see and the feelings that I wasn't - didn't belong here, you know, I belong back over there [in Iraq] (S1028).

In the narrative above, both lived experiences of trauma and feelings of not belonging emerge as having elicited problematic drinking in the context of post-deployment civilian life. Thus, participants described having binged on alcohol upon transitioning to the civilian arena and, free of institutional constraints, sometimes illicit substances, as well.

Some participants described habitual drinking as a strategy they used to psychologically and emotionally distance themselves from combat-related trauma. In this respect, one Veteran recalled how he was ready to party and just to get drunk after returning home from Iraq to dis- tance himself from the structural distress experienced during combat deployment: I was frustrated, he recounted. I lost friends; saw innocent kids get blown up in a bus, he explained. I just wanted to get away from that. I just wanted to get drunk and spend all my money (S1004).

Other participants described having consumed alcohol habitually in response to the lingering effects of physical trauma in the civilian context. For example, one 50-yearold Veteran drank habitually during each of three deployments, subsequently reproducing that routine practice by drinking routinely until inebriated in the civilian arena. $I$ drink by myself, he stated. That's what I used to do when my back was hurting or something to take my mind off it (i.e., the pain) (S1039). Thus, the participant described drinking as a way to cope with physical trauma during deployment (i.e., that's what I used to do when my back was hurting) which was reproduced as routine practice as a civilian (i.e., I drink by myself).

As drinking had been embodied as routine practice during military service and was furthermore routine in the context of the military, Veterans who habitually consumed alcohol in large quantities often did not immediately recognize how problematic this behavior was in a very different post-deployment context. The way in which drinking was both a routine practice elicited by distress and obfuscated by an internal habitus of perceptions is apparent in the following statement from a Veteran who had served overseas in Ecuador and Afghanistan:

And it's like they (i.e., the military) turn on a switch, but they don't turn back off the switch. But then when I got home, I didn't even realize, you know, that I had a [drinking] problem. I came home and I wasn't even home 24 hours and I was drinking! And I wasn't drinking once a week. I was drinking every day. It was a downhill battle from day one (S1034).

Echoing his words, a female Veteran of Operation Desert Storm described how an internal habitus embodied through socialization in the military structured her perception regarding what constitutes acceptable levels of alcohol consumption in a very different context. She stated that:

It's okay, you know, to be an alcoholic [in the military], because they're not realizing that it is a problem. And then you trying to live this everyday life without the military, and you're sitting at home - you can't function because you've got to drink all day, you know? You got issues because you're not in the military anymore and you're trying to medicate it, and so you think it's okay, but it's not okay (S1019).

Participants thus described a disjuncture between a habitus in which drinking was routine and the civilian 
arena in which such behavior was more often perceived as problematic, or hysterisis. Participants often described the consequences of habitual drinking out-of-context upon re-integration. For example, a Veteran deployed to Beirut, Granada and the Honduras who had lost a couple of jobs from drinking post-deployment recalled that: When I first got out [of the service], I got into fights all the time. All the time. I was working at a penitentiary up there, too and I got to shooting at people and stuff. He furthermore recounted how drinking as routine practice post-deployment had disrupted his marriage: I'd go out and I wouldn't come home. I started drinking really heavily, and after 16 years, my wife said 'I just can't take this no more' because it wouldn't stop (S1039). Such passages illustrate how reproducing once normative practices out-of-context resulted in a loss of capital in participants' civilian lives.

As all Veterans screened positive for alcohol misuse in order to participate in our study, their narratives often revealed promising lives come undone because of a socially valued and institutionally sanctioned practice embodied during military service and reproduced during re-integration. For some participants, drinking as routine practice outside the context of the military had devastating consequences, as it no longer resulted in the accrual of either social or economic capital. In another example, one Veteran who had been deployed to Afghanistan recounted how:

I didn't realize I had a [drinking] problem until two years later (i.e., after leaving the military). It's that so many things happened. I lost my house. I owned a house before I went over there [to Afghanistan]. And I lost my family. I lost my kid. I mean, you name it - it happened (S1034).

As vividly illustrated by the narratives above, once embodied as an adaptive strategy, the routine practice of drinking was reproduced habitually in response to distress evoked by structural conditions present in the context of civilian life. Removed from a social arena in which it was adaptive and rewarded, the routine practice of drinking became highly problematic, resulting in a loss of social and/or economic capital. Thus, not only was habitual drinking out-of-context post-deployment, it was often deleterious to re-integration for our participants.

\section{Discussion}

During substance use life history interviews, Veterans made sense of their problems with alcohol and difficulties re-integrating vis-à-vis institutional norms, shared beliefs about drinking, and social values and expectations learned in the context of military service. Participants described drinking as a routine - even expected - behavior in the context of life on military bases at home and abroad. They also repeatedly recounted how the very ordinariness of drinking in the military obscured a potentially deleterious practice that might, in other contexts, have been condoned and punished rather than tolerated (by superiors) and rewarded (by peers). Although reflecting the experiences of a subset of Veterans who began drinking excessively while in the military and reproduced that habit during reintegration, these narratives lend support to the assertion by Fuehrlein et al. ${ }^{27}$ that: Heavy drinking may often be normalized in military culture and such normalization may increase the likelihood that vulnerable veterans will develop alcohol use disorder.

Interpreting Veterans' substance use trajectories through the lens of practice theory lends fresh insights into alcohol misuse by Veterans. Our analysis revealed drinking as an adaptive strategy that was, for many Veterans in our study, initially embodied to accrue capital and subsequently employed to adapt to structural conditions which placed them at physical, emotional, and psychological risk during deployment. Drinking allowed participants to numb the unpleasant thoughts and emotions evoked by structural conditions while maintaining the appearance of physical and emotional toughness and selfcontrol that are so highly valued in the military. This illusion likely protected them from the negative consequences of violating social expectations and institutional norms. It also allowed participants to exert their agency over internal (i.e., distress) and external (i.e., social expectations) conditions present in the military context.

As deployment is time-limited, consuming alcohol often helped participants manage structural distress successfully in the short term, with few negative consequences. However, having learned to uncritically use alcohol to adapt to structural distress subsequently elicited this practice out-of-context post-deployment. The mismatch between an internal habitus in which drinking was normative and the social arena Veterans encountered in their lives as civilians resulted in hysteresis. As a result of this mismatch, a once-adaptive behavior was out-of-context, and therefore constrained participants' agency to reintegrate post-deployment. Practice theory thus rendered explicit, for the first time, the implicit cultural and social processes by which drinking habits learned during military service may be reproduced by Veterans.

To be clear, we do not claim that participants had no choice but to respond to distressing conditions by drinking excessively. On the contrary, social actors deliberately and strategically internalize practices that help them adapt to structural conditions. Problematic drinking is furthermore a complex phenomenon at the intersection of many lived experiences, such as genetics and gender, that did not emerge from our analysis. Rather than objective facts, our findings thus reflect participants' subjective sense of how their problems developed. Their biographies suggest that embodying drinking as routine practice in the military arena predisposed them to uncritically reproduce this behavior in response to distress encountered as civilians. 
Thus, routines embodied during military service constrained participants' ability to choose healthier strategies for managing distress. After all, individuals can only practice habits which they have learned.

Using a Bourdieusian framework to explicate why participants reproduced problematic drinking habits postdeployment aligns with recent work using practice theory to explain the re-integration of Veterans into civilian life. ${ }^{28}$ In this research, the authors described the distress that Veterans from the United Kingdom experienced during the difficult transition from a military to civilian habitus, and how that impacted their reintegration. Veterans in our study likewise described distress evoked by conditions present in the civilian context and how they consumed alcohol to cope with that distress. Together, these studies reveal reintegration as a vulnerable period for some Veterans that may require intervention.

Findings from our study furthermore provide important context for quantitative evidence correlating substance misuse among Veterans with PTSD, negative emotionality and depression, ${ }^{29}$ survivor's guilt,${ }^{30}$ combatrelated physical trauma ${ }^{1}$ and military sexual trauma. ${ }^{31}$ Participants described these problems as having been rooted in the structural conditions present in the military context. We have labelled the cognitive and emotional distress evoked by these structural conditions structural distress, thereby firmly situating conditions such as PTSD, moral injury, and survivor's guilt within a Bourdieusian framework. Moreover, using passages from the interviews to illustrate how structural distress was embodied as lived experiences of PTSD, survivor's guilt, and moral injury expands the scope of practice theory to explain the development of trauma-related disorders.

Healthcare providers should understand that drinking excessively is, for many Veterans, a learned behavior that was rewarded and valorized during military service. As consuming alcohol likely initially helped some former military personnel who subsequently develop unhealthy drinking habits cope with structural conditions during deployment, healthcare providers could explicitly acknowledge the adaptive purpose that drinking once served. Establishing rapport in this way could help providers to work with Veterans to help them recognize the mismatch between context and habitus which renders drinking excessively out-of-context in their lives as civilians, and thus no longer results in an accrual of capital.

Findings regarding the role played by alcohol in managing structural distress, combined with participants' accounts of how norms and shared understandings in the military habitus obfuscated the negative consequences of excessive drinking, suggest that multi-faceted treatment approaches for alcohol misuse will be most effective. For Veterans, engagement in evidence-based treatments (i.e., cognitive behavioral therapy), which help people embody healthy strategies for coping with the distressing thoughts and emotions which trigger substance use could be en- hanced by concurrently delivered group therapies in which peers share their experiences and support one another through treatment. However, it is worth noting that routinely drinking to excess was embodied while Veterans were still in the military. As such, the most effective interventions are likely to be those which target active duty personnel and are proactive, rather than reactive, occurring early in the military socialization process. Interventions aimed at building psychological resilience constitute pro-active strategies. Psychological resilience generally refers to the inter- and intra-personal process of adapting to and coping with stress and adverse life experiences (i.e., trauma). Resilience is multi-faceted, encompassing factors at the individual- (e.g., positive coping behaviors, affect and thinking, behavioral control), family- (e.g., emotional ties, communication, support, adaptability), and community-level (e.g., cohesion, collective efficacy). In the military context, resilience also includes unit-level factors (i.e., positive command climate, teamwork, cohesion). ${ }^{32}$

Many factors associated with resilience, such as coping, behavioral control, and adaptability, align well with the Bourdieusian concepts which emerged from analysis of the in-depth interviews, suggesting that resiliencebased interventions would resonate with active and former military personnel. Interventions that build resilience furthermore emphasize prevention rather than treatment and thus encourage the embodiment of routine practices early in the military socialization process, which our findings indicate is critical if the goal is to reduce problematic drinking and improve re-integration experiences. There thus exists a close fit between practice theory, which aligned with participants' own understanding of how their problematic drinking developed and their reintegration experiences, and resilience-based interventions.

The U.S. military has already begun implementing resilience training as part of a broader strategy aimed at increasing access to mental health services. These programs include Defenders Edge, implemented by the U.S. Air Force, Comprehensive Soldier Fitness and Battlemind, implemented by the U.S. Army, and Life Guard, implemented by the National Guard. ${ }^{33}$ As in the present study Veteran participants explicitly described having consumed alcohol to manage structural distress, such programs may indirectly reduce problematic drinking by providing healthier ways to cope with unpleasant thoughts and emotions. Evidence that psychological resilience may reduce excessive drinking in former military personnel comes from a recent study in which higher levels of psychological resilience correlated with lower rates of problematic drinking in Iraq and Afghanistan Veterans exposed to combat. ${ }^{34}$

Although initial evidence suggests that enlisted military personnel perceive interventions which build psychological resilience as helpful, ${ }^{33}$ a recent review of resilience training for active duty military personnel found wide- 
ranging differences in treatment effectiveness. ${ }^{35}$ Findings from this review indicate that a lack of leadership support, logistical problems, limited funding, poor fit with military culture, and mental health stigma have hampered the effectiveness of these programs. ${ }^{35}$ Situating these findings in relation to results from our study suggests that future research is needed to explore the adaptive practices of active duty military personnel who chose not to embody habitual drinking to identify potential resilience-enhancing habits aligned with the masculine-warrior habitus. This formative research would ensure that interventions aimed at building resilience are acceptable, and evidence based, potentially reducing some barriers to program effectiveness. Our findings indicate that it may also be necessary to adapt resilience programs to the different structural conditions encountered during military service (e.g., basic training, deployment, post-deployment) and to deliver these interventions longitudinally to achieve maximum effectiveness. As elucidated by practice theory, the success of such programs will also require a broader cultural shift in the military whereby less problematic coping and social behaviors are rewarded and valorized by peers, valued and encouraged by superiors, and receive institutional support in the form of funding.

\section{Conclusions}

Framing participants' biographies via practice theory supported the development of a hypothesis relating military culture to problematic drinking by Arkansas Veterans. During substance use life history interviews, Veterans described how social expectations, shared understandings and institutional norms in the military elicited drinking as habitual practice. Participants furthermore described this socially and institutionally patterned behavior as having been strategically used to manage structural distress during deployment on military bases overseas, often successfully and with few negative consequences. The deleterious consequences of a once adaptive practice reproduced uncritically out-of-context, however, were evident in poignant narratives in which participants described their difficulties re-integrating and living life as civilians. Qualitative findings revealing the way in which practices embodied and rewarded during military service can be uncritically reproduced out-of-context suggest that interventions aimed at teaching new recruits healthy coping strategies could, over time, reduce rates of harmful alcohol use by U.S. Veterans.

\section{References}

1. Barlas F, Higgins W, Pflieger J, et al. 2011 Health related behaviors survey of active duty military personnel [Internet]. Fairfax, VA: ICF International, Inc.; 2013. Available from: https://apps.dtic.mil/dtic/tr/fulltext/u2/a582287.pdf

2. Bray RM, Hourani LL, Rae KL, et al. 2002 Department of
Defense survey of health related behaviors among military personnel [Internet]. Research Triangle Park, NC: Research Triangle Institute; 2003. Available from: https://apps. dtic.mil/dtic/tr/fulltext/u2/a431566.pdf

3. Mattiko MJ, Olmsted KLR, Brown JM, et al. Alcohol use and negative consequences among active duty military personnel. Addict Behav 2011 36:608-14.

4. Ames GM, Cunradi CB, Moore RS, et al. Military culture and drinking behavior among U.S. Navy careerists. J Stud Alcohol Drugs 2007;68:336-44.

5. Gibbs DA, Rae Olmsted KL, Brown JM, et al. Dynamics of stigma for alcohol and mental health treatment among Army soldiers. Mil Psychol 2011;23:36-51.

6. Mash HBH, Fullerton CS, Ng THH, et al. Factor analysis of the Drinking Motives Questionnaire in a young adult U.S. Army sample. Psychol Rep 2014;115:339-50.

7. Ames GM, Duke MR, Moore RS, et al. The impact of occupational culture on drinking behavior of young adults in the U.S. Navy. J Mix Methods Res 2009;3:129-50.

8. Larson MJ, Wooten NR, Adams RS, et al. Military combat deployments and substance use: review and future directions. J Soc Work Pract Addict 2012;12:6-27.

9. Shirvani M, Reed MB, Clingan S. The relationship between emerging adult alcohol consumption and military enlistment. Mil Med 2017;182:e1731-7.

10. Derefinko KJ, Hallsell TA, Isaacs MB, et al. Substance use and psychological distress before and after the military to civilian transition. Mil Med 2018;183:e258-65.

11. Teeters J, Lancaster C, Brown D, et al. Substance use disorders in military veterans: prevalence and treatment challenges. Subst Abuse Rehabil 2017; Volume 8:69-77.

12. Calhoun PS, Elter JR, Jones ER, Kudler H, et al. Hazardous alcohol use and receipt of risk-reduction counseling among U.S. veterans of the wars in Iraq and Afghanistan. J Clin Psychiatry 2008;69:1686-93.

13. Bourdieu P. Distinction: A social critique of the judgement of taste. Cambridge, MA: Hardvard University Press; 1984.

14. Bourdieu P. The logic of practice. Stanford, CA: Stanford University Press; 1990.

15. Frohlich KL, Corin E, Potvin L. A theoretical proposal for the relationship between context and disease. Sociol Health Amp Illn 2001;23:776-97.

16. Cooper L, Caddick N, Godier L, et al. Transition from the military into civilian life: an exploration of cultural competence. Armed Forces Soc 2016;44:156-177.

17. Wacquant L. Pierre Bourdieu. In: Stones R, editor. Key sociological thinkers [Internet]. London: Macmillan Education UK; 1998 [cited 2020 Mar 25]. p. 215-29. Available from: http://link.springer.com/10.1007/978-1-349-26616-6_17

18. Higate P, ed. Military masculinities: Identity and the state. Westport, CT: Praeger Publishers; 2003.

19. Handwerker P. Quick ethnography. New York: AltaMira Press; 2001.

20. Vindrola-Padros C, Vindrola-Padros B. Quick and dirty? A systematic review of the use of rapid ethnographies in healthcare organisation and delivery. BMJ Qual Saf 2018;27:321-30.

21. Bernard HR, Wutich A, Ryan G. Analyzing qualitative data: systematic approaches. 2nd ed. Newbury Park, CA: Sage Publications, Inc; 2016.

22. Sheehan D, Janavs J, Knapp E, et al. Mini-International Neuropsychiatric Interview clinician rated, version 4.0. Tampa, FL: University of South Florida College; 1992. 
23. Cole AL, Knowles JG, editors. Lives in context: the art of life history research. New York: AltaMira Press; 2001.

24. Hubbard G. The usefulness of indepth life history interviews for exploring the role of social structure and human agency in youth transitions. Sociol Res Online 2000;4:102-13.

25. Hsieh H-F, Shannon SE. Three approaches to qualitative content analysis. Qual Health Res 2005;15:1277-88.

26. Birks M, Chapman Y, Francis K. Memoing in qualitative research: probing data and processes. J Res Nurs 2008;13:68-75.

27. Fuehrlein BS, Kachadourian LK, DeVylder EK, et al. Trajectories of alcohol consumption in US military veterans: results from the National Health and Resilience in Veterans Study. Am J Addict 2018;27:383-90.

28. Cooper L, Caddick N, Godier L, et al. A model of military to civilian transition: Bourdieu in action. J Mil Veteran Fam Health 2017;2:53-60.

29. Brady LL, Credé M, Harms PD, et al. Meta-analysis of risk factors for substance abuse in the US military. Mil Psychol 2019;31:450-61.

30. Campise RL, Geller SK, Campise ME. Combat Stress. In:
Kennedy CH, Zillmer EA, editors. Military psychology: clinical and operational applications. New York: Guilford Press; 2006. pp. 215-240.

31. Suris A, Lind L. Military sexual trauma: a review of prevalence and associated health consequences in veterans. Trauma Violence Abuse 2008;9:250-69.

32. Meredith LS, Sherbourne CD, Gaillot S. Promoting Psychological Resilience in the US Military; 2011. Santa Monica, CA: RAND Corporation; 2016.

33. Zinzow HM, Britt TW, McFadden AC, et al. Connecting active duty and returning veterans to mental health treatment: Interventions and treatment adaptations that may reduce barriers to care. Clin Psychol Rev 2012; 32:741-53.

34. Green KT, Beckham JC, Youssef N, et al. Alcohol misuse and psychological resilience among US Iraq and Afghanistan era veterans. Addict Behav 2014;39:406-13.

35. Thompson SR, Dobbins S. The applicability of resilience training to the mitigation of trauma-related mental illness in military personnel. J Am Psychiatr Nurses Assoc 2018;24:23-34. 